\title{
The Accumulative Effect of Concentric-Biased and Eccentric- Biased Exercise on Cardiorespiratory and Metabolic Responses to Subsequent Low-Intensity Exercise: A Preliminary Study
}

\author{
by \\ James Peter Gavin ${ }^{1,2}$, Stephen Myers ${ }^{1}$, Mark Elisabeth Theodorus Willems ${ }^{1}$
}

The study investigated the accumulative effect of concentric-biased and eccentric-biased exercise on cardiorespiratory, metabolic and neuromuscular responses to low-intensity exercise performed hours later. Fourteen young men cycled at low-intensity ( 60 rpm at 50\% maximal oxygen uptake) for $10 \mathrm{~min}$ before, and $12 \mathrm{~h}$ after: concentric-biased, single-leg cycling exercise (CON) (performed $~ 19: 30 h$ ) and eccentric-biased, double-leg knee extension exercise (ECC) ( 06:30 h the following morning). Respiratory measures were sampled breath-by-breath, with oxidation values derived from stoichiometry equations. Knee extensor neuromuscular function was assessed before and after CON and ECC. Cardiorespiratory responses during low-intensity cycling were unchanged by accumulative CON and ECC. The RER was lower during low-intensity exercise $12 \mathrm{~h}$ after CON and ECC $(0.88 \pm 0.08)$, when compared to baseline $(0.92 \pm 0.09 ; p=0.02)$. Fat oxidation increased from baseline $\left(0.24 \pm 0.2 \mathrm{~g} \cdot \mathrm{min}^{-1}\right)$ to $12 \mathrm{~h}$ after CON and ECC $\left(0.39 \pm 0.2 \mathrm{~g} \cdot \mathrm{min}^{-1} ; p=0.01\right)$. Carbohydrate oxidation decreased from baseline $\left(1.59 \pm 0.4 \mathrm{~g} \cdot \mathrm{min}^{-1}\right)$ to $12 \mathrm{~h}$ after CON and ECC $\left(1.36 \pm 0.4 \mathrm{~g} \cdot \mathrm{min}^{-1} ; p=0.03\right)$. These were accompanied by knee extensor force loss (right leg: $-11.6 \%, p<$ 0.001; left leg: $-10.6 \%, p=0.02$ ) and muscle soreness (right leg: $2.5 \pm 0.9, p<0.0001$; left leg: $2.3 \pm 1.2, p<0.01$ ). Subsequent concentric-biased and eccentric-biased exercise led to increased fat oxidation and decreased carbohydrate oxidation, without impairing cardiorespiration, during low-intensity cycling. An accumulation of fatiguing and damaging exercise increases fat utilisation during low intensity exercise performed as little as $12 \mathrm{~h}$ later.

Key words: muscle damage, eccentric exercise, exercise metabolism, low-intensity exercise, substrate oxidation.

\section{Introduction}

Unaccustomed, high-intensity resistance exercise is associated with symptoms of exerciseinduced muscle damage, particularly when involving eccentric contractions. The high eccentric forces produced during resistance exercise often result in acute force loss, muscle soreness and heightened effort perception during habitual activities in the subsequent hours and days. These persisting symptoms can impair endurance performance for lower- (Asp et al., 1998; Sargeant and Dolan, 1987) and upper-body exercise (Doncaster and Twist, 2012).
Understanding the potential relationships between the physiological mechanisms of muscle damage is important to devise prevention and recovery strategies. A common approach to alleviate muscle damage symptoms after heavy training bouts is to prescribe low-intensity activity (Ahmaidi et al., 1996; Suzuki et al., 2004), aimed to improve muscle microcirculation.

Eccentric contractions may alter cardiorespiratory function during exerciseinduced muscle damage, yet greater oxygen cost following duathlon (Calbet et al., 2001) and

1 - Department of Sport and Exercise Sciences, University of Chichester, United Kingdom.

2- Department of Sport and Physical Activity, Bournemouth University, United Kingdom. 
marathon events (Kyrolainen et al., 2000) suggests concentric-biased contractions also exert substantial ventilatory impairment. Elsewhere, bench-stepping exercise, involving both concentric and eccentric contractions, has resulted in elevated minute ventilation ( $\dot{\mathrm{V}} \mathrm{E})$, the respiratory exchange ratio (RER), and creatine kinase levels when cycling (15 $\mathrm{min}$, at $80 \%$ maximal rate of oxygen uptake (ن்2max)) $48 \mathrm{~h}$ later (Gleeson et al., 1995). However, oxygen uptake ( $\dot{\mathrm{V} O 2)}$ was unaffected. Similar results have been observed at lower cycling workloads (60 and $80 \%$ peak $\dot{V}$ O2), with one-hundred countermovement jumps increasing perceptual and ventilatory responses, as well as impairing timetrial performance (Twist and Eston, 2009). The relationship between $\dot{V} E$ and perceived effort appears intensity-dependent, with the greater ventilatory responses at higher cycling workloads accompanied by higher perceived effort, $48 \mathrm{~h}$ after squatting exercise (Davies et al., 2009). Disrupted muscle microcirculation, occurring during exercise-induced muscle damage (Kano et al., 2005) elevates $\dot{V} E$ for subsequent exercise (Haouzi et al., 2004). A shortfall in oxygen delivery and utilisation may disturb muscle metabolism resulting in a shift to anaerobic metabolism, typified by increased lactate production (Braun and Dutto, 2003; Gleeson et al., 1995) and an emerging $\dot{\mathrm{VO}} 2$ slow component at higher exercise intensities (Davies et al., 2008). It appears activity involving repeated, concentric and eccentric contractions may increase relative exercise-stress, and therefore influence exercise intensity and performance. This is important for recovery, when competing and/or training over repeated days, and may also present a novel strategy to manipulate substrate utilisation for subsequent exercise bouts.

Cardiorespiratory, metabolic and perceptual responses to exercise are intensitydependent, as shown at moderate- (Molina and Denadai, 2011), heavy- (Schneider et al., 2007) and severe-intensities (Davies et al., 2009), as well as all-out effort (Twist and Eston, 2009). To-date, cardiorespiratory and metabolic responses for low-intensity exercise (at 50\% $\dot{\mathrm{V} O 2 m a x), ~}$ following exercise involving repeated, concentric and eccentric contractions have not been studied. Greater understanding of physiological responses during low-intensity exercise, with accompanying muscle fatigue and damage has relevance to i) athletes exercising for recovery, ii) those undertaking exercise rehabilitation and exerciseintolerant clinical groups, and iii) individuals aiming to manipulate substrate metabolism. For example, individuals using concurrent resistance exercise for training, and low-intensity aerobic exercise for recovery, would benefit from the knowledge of the acute effects of muscle fatigue and damage. Although $12 \mathrm{~h}$ after, is very acute for muscle damage, findings will provide insight into how early potential cardiovascular, respiratory and metabolic changes may emerge. In application, this is important to athletes performing morning training and low-intensity, evening training (such as swimmers, triathletes or track athletes), as well as overweight individuals targeting enhanced fat oxidation. Therefore, the study aim was to investigate the accumulative effect of concentric-biased and eccentric-biased exercise on cardiorespiratory and metabolic responses to low-intensity exercise in the hours after. The secondary aim was to examine the accompanying neuromuscular properties of the exercising muscle group, the knee extensors.

\section{Material and Methods}

\section{Participants}

Fourteen healthy men (mean \pm SD; age, 22 \pm 3 years, body height, $179 \pm 6 \mathrm{~cm}$, body mass, 76.4 $\pm 15.0 \mathrm{~kg}$, body fat, $9.2 \pm 2.5 \%$ ) provided written informed consent to partake in the study. None had musculoskeletal and joint injury, all were recreationally active university students (exercise frequency, $2.9 \pm 1.3$ days per week) and not undertaking any form of lower-body resistance training during the testing period. Sample size estimation was based on data for MVC force loss $(-19.8 \%, \alpha$ level $=0.05$, power $=0.80(1-\beta)$ (Faul et al., 2007). The research protocol was approved of by the University of Chichester Research Ethics Committee and conducted according to the Declaration of Helsinki.

\section{Procedures}

Participants completed the following four experimental sessions: i) a familiarisation, ii) concentric-biased, single-leg cycling exercise $(\mathrm{CON})$, iii) eccentric-biased, double-leg knee extension exercise (ECC), and iv) $12 \mathrm{~h}$ post lowintensity cycling. Single-leg cycling was 
performed to pre-fatigue the exercising leg, with concentric actions as part of a larger study (Gavin et al., 2015). Cardiorespiratory and metabolic responses were measured during $10 \mathrm{~min}$ of lowintensity, constant-load cycling prior to session two, and during session four. Responses were only measured $12 \mathrm{~h}$ post i) to examine a potential early-onset muscle damage, metabolic response and ii) to avoid influencing neuromuscular recovery as part of a related study (Gavin et al., 2015). Participants were instructed to refrain from heavy physical activity throughout the testing period, and to not to consume alcohol $24 \mathrm{~h}$ before each session. The familiarisation involved an incremental cycling trial to assess $\dot{\mathrm{V}}_{2 \max }$ and practice single-leg cycling. Session two occurred in the evening, at least $48 \mathrm{~h}$ later, and involved exhaustive, single-leg cycling in a $3 \mathrm{~h}$ fasted state, with the right leg (participant leg dominance: twelve (86\%) right leg; two (14\%) left leg). This was preceded by the two-leg, low-intensity cycling, a low carbohydrate evening meal ( 3932 kJ: protein $\sim 960 \mathrm{~kJ}$, carbohydrate $\sim 70 \mathrm{~kJ}$, fat 2907 $\mathrm{kJ}$ ) of three eggs and six bacon slices, and then a breakfast of two eggs the next day (Steensberg et al., 2002). Session three occurred early the following morning, and involved 100 single-leg, maximal eccentric knee extensions. Session four involved a $12 \mathrm{~h}$ retest of two-leg, low-intensity cycling exercise. Water was provided ad libitum during all sessions.

\section{Familiarisation}

After collection of anthropometric data, subjects performed an incremental cycling test to volitional exhaustion on an electronically controlled ergometer (Excalibur Sport 925900, Lode, Groningen, the Netherlands). Participants cycled at a $\sim 75 \mathrm{rpm}$ cadence at $80 \mathrm{~W}$ for $60 \mathrm{~s}$, with $30 \mathrm{~W}$ increments every $60 \mathrm{~s}$, until a cadence of 50 rpm could not be maintained (Heil et al., 1995). The test measured the individual's power at $\dot{\mathrm{V}}_{2 \max }$ which was used to determine workload for i) the low intensity cycling and, ii) the CON.

\section{Cardiorespiratory, metabolic and neuromuscular} measures

Prior to CON and $12 \mathrm{~h}$ after ECC, two-leg low-intensity cycling was performed for $10 \mathrm{~min}$ at $\sim 60 \mathrm{rpm}$ on a friction-braked ergometer (Monark Ergomedic 824E, Varberg, Sweden) at 50\% $\dot{\mathrm{VO}}_{2 \max }$ $(1.5 \pm 0.2 \mathrm{~kg} ; 116 \pm 17 \mathrm{~W})$. Participants were instructed to sustain a moderate-carbohydrate version of their habitual diet during the experimental period. Dietary information packs were provided to inform food preferences, and participants maintained food diaries which were monitored by the investigator on each visit. Respiratory responses were sampled throughout using a portable metabolic cart (Cosmed $\mathrm{K}_{4} \mathrm{~b}^{2}$, Rome, Italy), with the heart rate (HR) measured continuously (Polar Electro Oy, Kempele, Finland). The metabolic cart was pre-calibrated to atmospheric air and gases of known concentration $\left(\mathrm{O}_{2}\right.$ concentration, $15.12 \% ; \mathrm{CO}_{2}$ concentration, $5.03 \%)$. The turbine flow meter was calibrated with a 3 L calibration syringe (Model 5570, Hans Rudolph Inc., Kansas, USA) and with the investigator breathing at a regular, constant rhythm into a low-resistance face mask (Cosmed $\mathrm{K} 4 \mathrm{~b}^{2}$ User Manual). Breath-by-breath expired gas was collected during the low-intensity cycling, and averaged over $15 \mathrm{~s}$ periods for: tidal volume, $\dot{\mathrm{V}}_{\mathrm{E}}, \dot{\mathrm{V}} \mathrm{O}_{2}$, carbon dioxide output $\left(\dot{\mathrm{V}} \mathrm{CO}_{2}\right)$, the RER and the HR. According to recommendations by Jeukendrup and Wallis (2005) for low-intensity exercise ( 40 to $50 \% \dot{\mathrm{V}}_{2 \max }$ ), fat and carbohydrate oxidation values derived from respiratory measures $\left(\dot{\mathrm{V}}_{2}\right.$ and $\left.\dot{\mathrm{V}} \mathrm{CO}_{2}\right)$ were modified using the following equations:

Equation 1 Stoichiometry calculations proposed by Jeukendrup and Wallis (2005) for low-intensity exercise (40 to $50 \% \dot{\mathrm{V}}_{2 \max }$ ).

Fat oxidation $\left(\mathrm{g} \cdot \mathrm{min}^{-1}\right) \quad 1.695 \times \dot{\mathrm{VO}}_{2}-$ $1.701 \times \dot{\mathrm{V}} \mathrm{CO}_{2}-1.77 n$

\section{Carbohydrate oxidation $\left(\mathrm{g} \cdot \mathrm{min}^{-1}\right)=4.344 \mathrm{x}$ $\dot{\mathrm{V} C O}{ }_{2}-3.061 \times \dot{\mathrm{V}}_{2}-0.40 n$}

Where $\dot{\mathrm{VO}}_{2}$ and $\dot{\mathrm{V}} \mathrm{CO}_{2}$ are presented in $\mathrm{L} \cdot \mathrm{min}^{-1}$ and oxidation rates in $\mathrm{g} \cdot \mathrm{min}^{-1} ; n=$ nitrogen contribution was assumed negligible. Oxidation estimations were based upon the assumption that total carbohydrate oxidation was derived from $50 \%$ plasma glucose and 50\% muscle glycogen. Fat oxidation was derived from circulating longchain fatty acids, low-density lipoprotein triglycerides and intramuscular triglycerides (Romijn et al., 1993). However, indirect calorimetry did not allow us to determine the proportional contribution of each (Jeukendrup and Wallis, 2005).

Knee extensor neuromuscular function 
was assessed before and after CON and ECC on a custom-built, strength-measurement chair. Seated and secured at the hips and chest, participants were positioned with hip and knee joints at 1.57 rad. The ankle was attached to a steel chain leading to the chair base and a calibrated s-beam load-cell. A DS7A electrical stimulator and a NeuroLog pulse generator (Digitimer Limited, Welwyn Garden City, UK) delivered electrical stimulation through two saline treated electrodes $(9 \times 18 \mathrm{~cm})$ placed over the proximal and distal part of the thigh. A single, submaximal twitch was applied to the relaxed knee extensors. A warm up of three submaximal isometric knee extensions was followed by three MVC ( 3 to $5 \mathrm{~s}$, with $2 \mathrm{~min}$ rest). Muscle soreness was assessed with the muscle-belly palpated under contraction, using a visual analogue scale ( 0 , no pain; 10 , extreme pain). Verbal encouragement and forcetime feedback from the computer monitor were provided throughout maximal testing.

\section{Concentric-biased exercise}

Between 19:00 and 20:00 h participants performed single-leg cycling exercise on a customised cycle ergometer (Monark Ergomedic 824E, Varberg, Sweden). The left crank was removed, and replaced with a platform supporting the left leg in a relaxed position. The right foot was secured into the pedal of the right crank. Maximal single-leg cycling workload was equated as $74 \%$ of maximal two-leg workload (at $\dot{V} \mathrm{O}_{2 \max }$ ) from the familiarisation (Pernow and Saltin, 1971); all subsequent single-cycling workloads were derived from the single-leg maximal value and referred to hereafter. Singleleg cycling started at $75 \mathrm{rpm}$ with $20 \mathrm{~min}$ at $75 \%$ $\dot{\mathrm{V}} \mathrm{O}_{2 \max }(2.3 \pm 0.3 \mathrm{~kg} ; 178 \pm 27 \mathrm{~W})$, after which eight $90 \mathrm{~s}$ sprints were performed with a 1:1 work-torest ratio (workload decreased from 90 to $55 \%$ $\dot{\mathrm{V}}_{2 \max }$ at $5 \%$ decrements), before cycling at $85 \%$ $\dot{\mathrm{V}}{ }_{2 \max }$ to volitional exhaustion (Pilegaard et al., 2002).

\section{Eccentric-biased exercise}

The following morning $(\sim 06: 30 \quad \mathrm{~h})$ participants performed maximal, eccentric knee extensions with the right leg, and then the left leg. Ten sets of ten eccentric contractions were performed (60 s rests between each set) using a pre-calibrated isokinetic dynamometer (Humac Norm, Cybex, NY, USA) (Byrne et al., 2001).
Participants were seated and fastened in the strength testing chair with the hip joint at $1.57 \mathrm{rad}$. The lateral femoral epicondyle of the exercising leg was aligned to the gravity-corrected dynamometer and the ankle joint was secured to the lever arm. Participants were instructed to exert maximal force against the pre-programmed dynamometer lever arm, which moved at 1.57 $\mathrm{rad} \cdot \mathrm{s}^{-1}$ from full knee extension $(0 \mathrm{rad})$ to full flexion (1.74 rad). Standardised verbal encouragement was provided throughout, with instantaneous visual feedback of torque-time traces from the dynamometer interface.

\section{Statistical analysis}

Paired samples $t$-tests compared cardiorespiratory and metabolic responses for low-intensity cycling, and neuromuscular function, before and $12 \mathrm{~h}$ after CON and ECC (PASW Statistics 18.0, California, USA). Where data did not satisfy normal distribution (RER, fat oxidation and muscle soreness), Wilcoxon signed-rank tests were used. Cardiorespiratory and metabolic responses were compared for the final minute of low-intensity cycling, between responses before, and $12 \mathrm{~h}$ after CON and ECC. Cohen's Effect sizes were calculated for meaningful differences ( 0.2 for small, 0.5 for moderate, and 0.8 for large). Data are presented as mean $\pm \mathrm{SD}$, with a significance level of $p<0.05$ accepted.

\section{Results}

\section{Cardiorespiratory measures}

Cardiorespiration during low-intensity cycling was not affected by CON and ECC. Tidal volume was $1.58 \pm 0.3 \mathrm{~L}$ at baseline and $1.63 \pm 0.3$ $\mathrm{L} 12 \mathrm{~h}$ after ECC $(p=0.6, d=0.16) ; \dot{\mathrm{V}}_{\mathrm{E}}$ was $35.2 \pm$ 6.6 $\mathrm{L} \cdot \mathrm{min}^{-1}$ at baseline and $37.5 \pm 5.7 \mathrm{~L} \cdot \mathrm{min}^{-1}$ at $12 \mathrm{~h}$ after ECC $(p=0.3, d=0.36)$; $\dot{\mathrm{V}}_{2}$ was $1.46 \pm 0.3$ $\mathrm{L} \cdot \mathrm{min}^{-1}$ at baseline and $1.59 \pm 0.2 \mathrm{~L} \cdot \mathrm{min}^{-1} 12 \mathrm{~h}$ after ECC $(p=0.1, d=0.53) ; \dot{\mathrm{V} C O} 2$ was $1.36 \pm 0.2 \mathrm{~L} \cdot \mathrm{min}^{-1}$ at baseline and $1.38 \pm 0.2 \mathrm{~L} \cdot \mathrm{min}^{-1} 12 \mathrm{~h}$ after ECC $(p$ $=0.7, d=0.11) ; \mathrm{HR}$ was $109 \pm 16 \mathrm{~b} \cdot \mathrm{min}^{-1}$ at baseline and $111 \pm 14 \mathrm{~b} \cdot \mathrm{min}^{-1} 12 \mathrm{~h}$ after ECC $(p=0.1, d=$ 0.18 ).

\section{Substrate oxidation}

During low-intensity cycling, the RER was lower $12 \mathrm{~h}$ after ECC $(0.88 \pm 0.08)$, than at baseline (0.92 $\pm 0.09 ; p=0.02, d=0.51$; Figure 1). Fat oxidation was higher $12 \mathrm{~h}$ after ECC $(0.39 \pm 0.2$ $\left.\mathrm{g} \cdot \mathrm{min}^{-1}\right)$, than at baseline $\left(0.24 \pm 0.2 \mathrm{~g} \cdot \mathrm{min}^{-1} ; p=\right.$ 
$0.014, d=0.72$; Figure 2). Carbohydrate oxidation was lower $12 \mathrm{~h}$ after ECC $\left(1.36 \pm 0.4 \mathrm{~g} \cdot \mathrm{min}^{-1}\right)$, than at baseline $\left(1.59 \pm 0.4 \mathrm{~g} \cdot \mathrm{min}^{-1} ; p=0.03, d=0.61\right.$; Figure 3).

\section{Neuromuscular measures}

Twelve hours after ECC, knee extensor MVC decreased (right leg: $p=0.001,-11.6 \%, d=0.93$; left leg: $p=0.02,-10.6 \%, d=0.72)$ and soreness increased (right leg: $p=0.0001,2.5 \pm 0.9, d=1.56$; left leg: $p=0.008,2.3 \pm 1.2, d=0.99)$. The maximal rate of force decrease reduced after $\mathrm{CON}$ (right leg: only $p=0.05, d=0.67)$, but single twitch force parameters were not significantly altered after CON and ECC (Table 1).

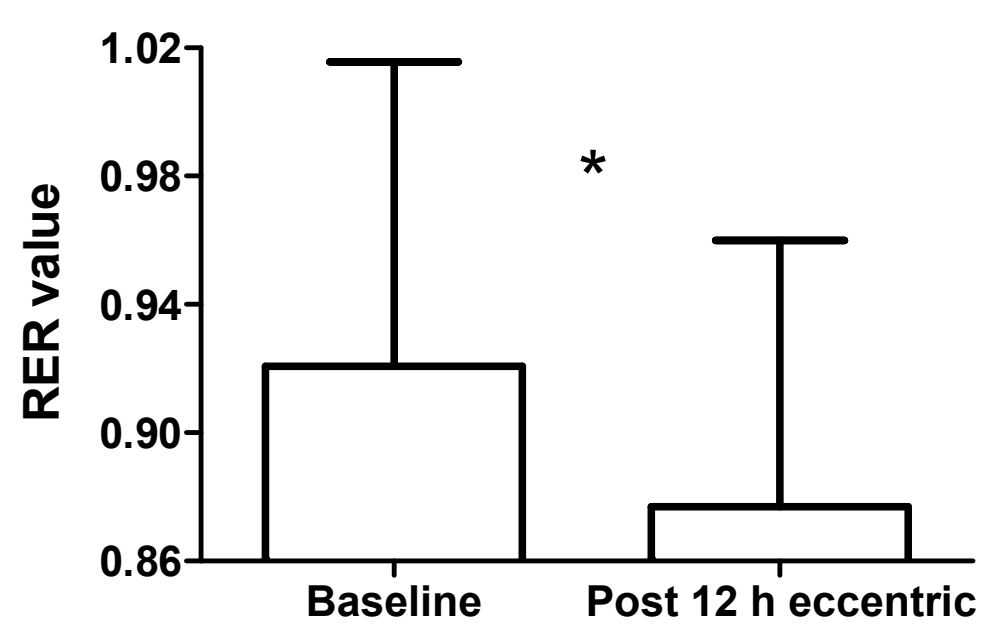

Figure 1

Respiratory exchange ratio (RER) values at baseline and $12 \mathrm{~h}$ after maximal, eccentric knee extensions with a prior concentric exercise.

* Significant difference between time points, $p<0.05$

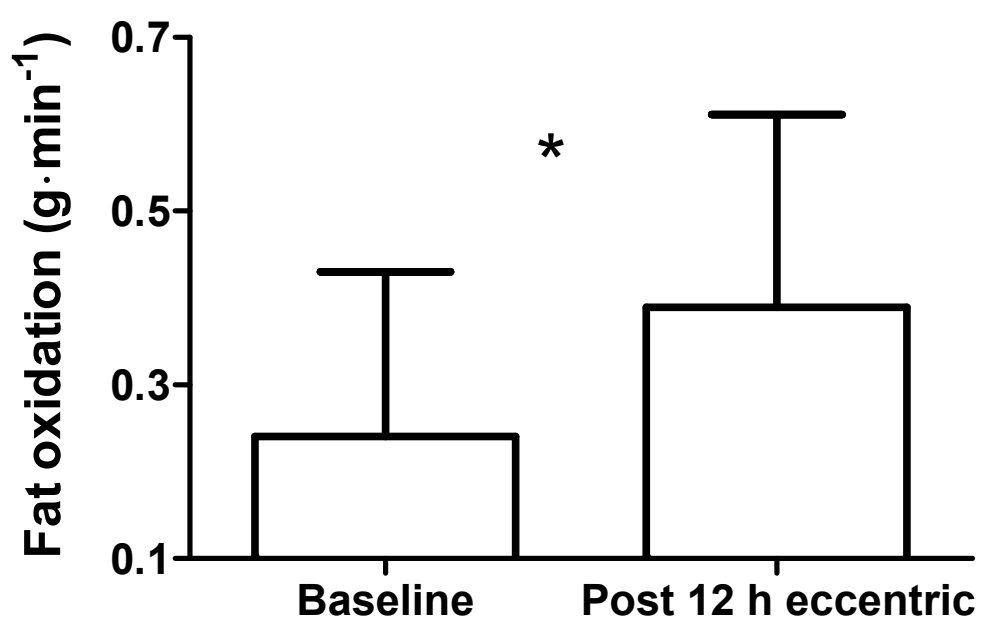

Figure 2

Fat oxidation values at baseline and $12 \mathrm{~h}$ after maximal, eccentric knee extensions with prior concentric exercise. Values are mean $\pm S D$.

* Significant difference between time points, $p<0.05$ 


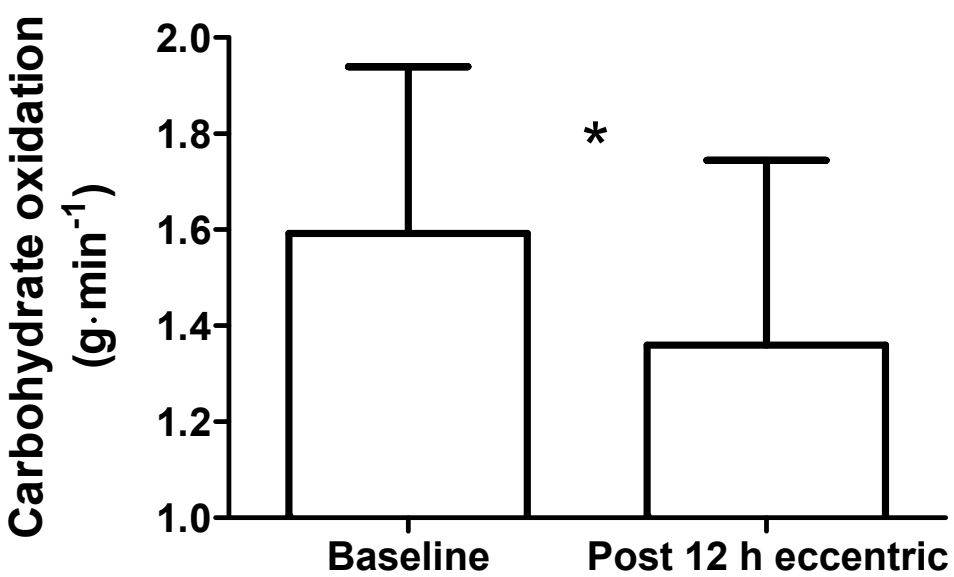

Figure 3

Carbohydrate oxidation at baseline and $12 \mathrm{~h}$ after maximal, eccentric knee extensions with prior concentric exercise. Values are mean $\pm S D$.

* Significant difference between time points, $p<0.05$

Table 1

Knee extensor contractile parameters (right and left legs) during an evoked single twitch before, and after concentric-biased (CON) and eccentric-biased (ECC) exercise

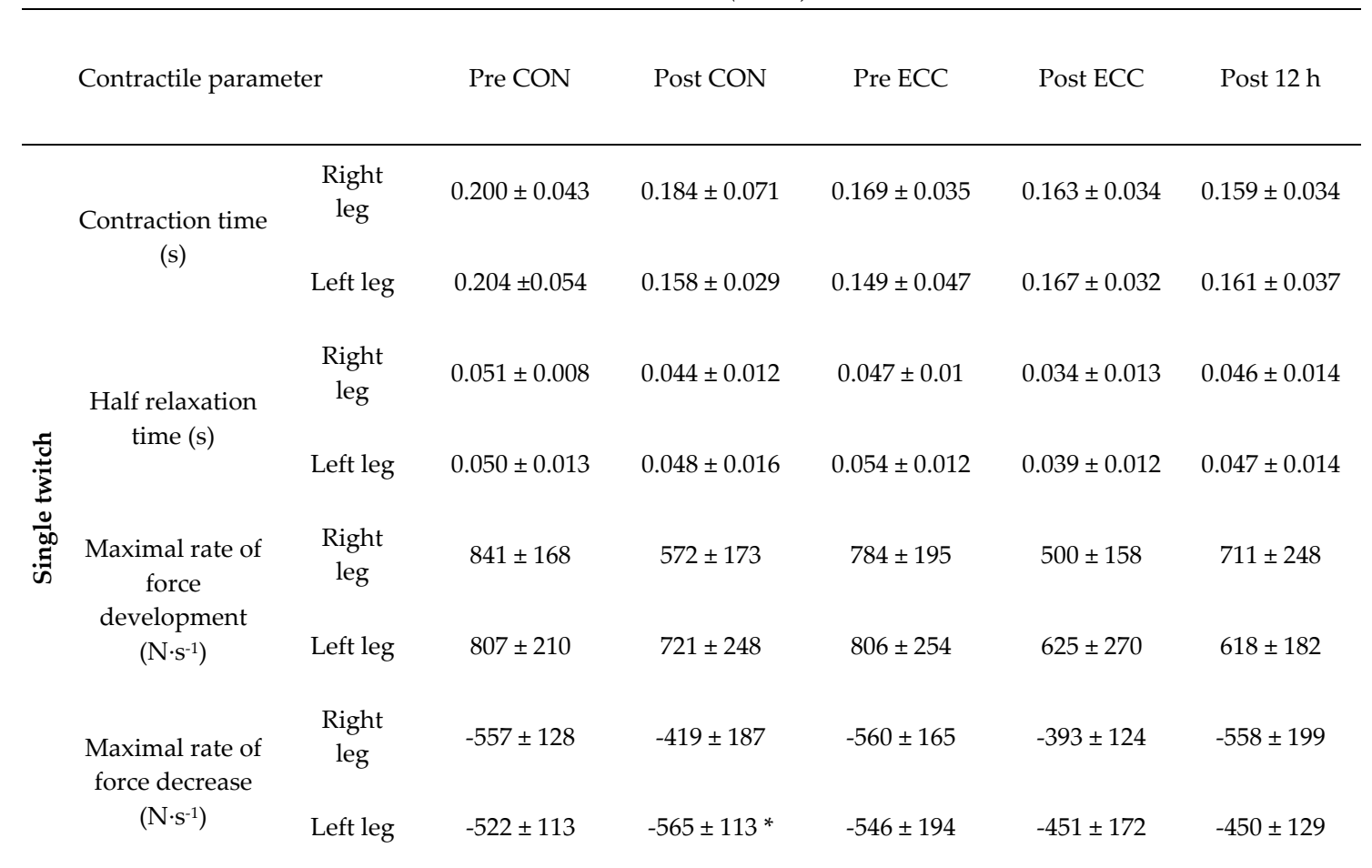

Data presented as mean $\pm S D$. * Significant difference between time points, $p<0.05$. 


\section{Discussion}

To characterise the relationship between cardiorespiratory and metabolic responses to exercise in a state of muscle damage, lowintensity cycling was performed before and after exhausting concentric, and then eccentric exercise. Our findings indicate that early mild-muscle damage of the knee extensors altered substrate metabolism, independent of cardiorespiratory measures, during low-intensity exercise. Peak damage occurs between 24 to $48 \mathrm{~h}$, however, force loss results $(-11.6 \%$ right leg; $-10.6 \%$ left leg) indicate mild damage at $12 \mathrm{~h}$ according to Paulsen et al.'s (2012) criteria ( $<20 \%$ force loss). Despite unchanged cardiorespiratory function, a reduced RER was supported by increased fat oxidation and decreased carbohydrate oxidation. We hypothesised that knee extensor force loss $12 \mathrm{~h}$ after eccentric exercise would be associated with increased ventilation during low-intensity exercise. If muscle damage was manifest at $12 \mathrm{~h}$, greater group III and IV afferent activation would increase ventilation. Force loss with muscle damage is associated with decreased cycling power output (Black and Dobson, 2012; Byrne et al., 2001) and treadmill running performance (Marcora and Bosio, 2007). Our eccentric exercise induced mild-muscle damage was evidenced by increased muscle soreness and force loss. Elsewhere, Davies et al. (2009) found similar force loss from squatting exercise after $30 \mathrm{~min}(-14 \%)$ and $48 \mathrm{~h}(-11 \%)$. We found the force loss during early muscle damage was not sufficient to alter effort perception for low-intensity cycling. Near peak muscle damage $(48 \mathrm{~h})$, Twist and Eston (2009) observed increased effort perception and $\dot{\mathrm{V}}$ /VंO2 (but unaltered $\dot{\mathrm{V} O} 2, \mathrm{HR}$ and RER) during constant-load, submaximal cycling. At the same time-point, reduced time-trial performance was associated with greater effort perception.

Evidence supporting altered cardiorespiratory function when exercising in a muscle damaged state remains equivocal (Chen et al., 2007; Gleeson et al., 1995). We found no change in tidal volume, $\dot{\mathrm{V}} \mathrm{E}, \dot{\mathrm{V}} \mathrm{O} 2, \dot{\mathrm{V}} \mathrm{CO} 2$ and $\mathrm{HR}$ during low intensity cycling $12 \mathrm{~h}$ after eccentric exercise, preceded by concentric exercise. This is not entirely surprising given previous mixed reports for greater exercise intensities, and with more severe eccentric exercise protocols. At higher cycling intensities, Davies et al. (2009) showed association between ventilation and effort perception for exercise above the gas exchange threshold. Participants underwent $6 \mathrm{~min}$ of moderate-, then severe-intensity cycling to volitional exhaustion, at baseline and $48 \mathrm{~h}$ preceding squatting exercise using $70 \%$ body weight. For moderate-intensity cycling, $\dot{\mathrm{VE}}$ increased from $34.5 \pm 5.0$ to $36.3 \pm 3.8 \mathrm{~L} \cdot \mathrm{min}^{-1}$ post $48 \mathrm{~h}$, which was not dissimilar to our own observation from $35.2 \pm 6.6$ to $37.5 \pm 5.7 \mathrm{~L} \cdot \mathrm{min}^{-1} 12$ $\mathrm{h}$ later. These authors cited relation between respiratory responses and perceived exertion at severe-, but not moderate-intensities. This may suggest an intensity dependence on the manifestation of muscle damage symptoms when examining cardiorespiratory responses. Unaltered VO2 response in this study may be due to predominant recruitment of type I muscle fibres, as opposed to type II fibres.

Steady-state measures, such as $\dot{\mathrm{V}} 2$ and the RER, can indicate energy demands at a given work rate, but tell us little about oxygen delivery and utilisation. Participants cycled at an intensity of $50 \% \dot{\mathrm{V} O} 2 \mathrm{max}$, which was sufficiently low that inter-individual differences in $\dot{\mathrm{VO}} 2$ and $\dot{\mathrm{V}} \mathrm{CO} 2$ were absent. Using the same isokinetic eccentric bout as our own, Molina and Denadai (2011) compared $\dot{\mathrm{V} O} 2$ kinetics of untrained males during moderate-intensity cycling at either 50 or $100 \mathrm{rpm}$ cadence. Cycling at $50 \mathrm{rpm}$, there was no change in phase II $\dot{\mathrm{VO}} 2$ kinetics. Yet, at $100 \mathrm{rpm}$, a delay in $\mathrm{V} O 2$ became apparent at $24 \mathrm{~h}$, suggesting exercise-induced impairment in oxygen delivery, in part, depends upon cadence. Awareness of the changes in cardiorespiratory and metabolic responses during the early-onset of muscle damage may impact the decisions of athletes, coaches and exercise scientists, when performing consecutive bouts of exercise. Such findings have importance for athletes including sailors, skiers and multi-event competitors, for whom it is common to perform repeat non-maximal/recovery exercise sessions over a single day.

The relative dietary contributions of carbohydrate and fat influence the RER at rest and during exercise. Interestingly, the RER decreased during low-intensity cycling in exercise-induced muscle damage at $12 \mathrm{~h}$, indicating increased fat utilisation for the same given work rate as 
baseline. However, it is plausible that glycogen reduction from the concentric-biased exercise attenuated this decrease. Osborne and Schneider (2006) previously found glycogen depletion reduced the RER (depleted: 0.97; normal: 1.06) when performing $8 \mathrm{~min}$ of heavy-intensity, constant-load cycling. Lima-Silva et al. (2009) also found greater fat oxidation for cycling exercise with reduced glycogen, at workloads above the second lactate turnpoint. Such metabolic alterations during exercise warrant further research, particularly the approach of using prior, mixed concentric and eccentric exercise to promote fat metabolism. Yamanaka and associates (2012) used three separate, $120 \mathrm{~s}$ intense-cycling bouts (100 to $105 \%$ VंO2peak), finding the RER decreased from bout one (1.05) to three (0.96). Therefore, our concentric-biased cycling exercise may have had a lasting effect on metabolic, but not cardiorespiratory responses. At baseline fat oxidation was $0.24 \mathrm{~g} \cdot \mathrm{min}^{-1}$, increasing to $0.39 \mathrm{~g} \cdot \mathrm{min}^{-1} 12 \mathrm{~h}$ into exercise-induced muscle damage. Whole-body, fat oxidation rates decline during high-intensity exercise $(85 \% \dot{\mathrm{V} O} 2 \mathrm{max})$ as a result of muscle glycogen becoming the primary energy source (Romijn et al., 1993). In addition, as exercise-induced muscle damage disrupts glucose mobilisation in the young (Kirwan et al., 1992), our finding of greater fat (and reduced carbohydrate) contribution for low-intensity exercise during exercise-induced muscle damage, agrees with those at higher intensities. Tuominen et al. (1996) used the term 'post marathon paradox', to describe their observation of increased fat oxidation (by 55\%) and decreased carbohydrate oxidation (by $-43 \%$ ) the morning following a marathon run. Similar to marathon running, which involves repeated, concentric and eccentric contractions of the knee extensors, the combination of our CON and ECC may partly explain the similar results.

Our experimental question was whether symptoms of exercise-induced muscle damage would become manifest for low-exercise intensity, in the following hours. This limited cardiorespiratory and metabolic measurement to an intensity below that of competitive and recreational activity, yet these findings have relevance to exercise during recovery. For example, bouts of fatiguing and damaging resistance exercise are often followed by low- intensity, aerobic exercise for recovery. The results also indicate that the accumulative effect of concentric and eccentric exercise elevates fat oxidation during acute, subsequent exercise. Optimising fat oxidation, this is relevant to i) encourage weight loss in the overweight/obese, and ii) act as a training stimulus for an athlete, that also promotes glycogen sparing. This study demonstrates that a protocol involving concentricbiased, single leg exercise, and then eccentricbiased, double leg exercise, did not alter cardiorespiratory responses during low-intensity cycling exercise, but did alter substrate oxidation. Respiratory measures remained unchanged following eccentric exercise, preceded by concentric-biased exercise; however, fat oxidation increased, while carbohydrate oxidation and the RER decreased. These findings suggest metabolic disruption may occur in exercise-induced muscle damage, without changing ventilation. Increased fat oxidation may be due to impaired insulin action with muscle damage; but this was not supported by cardiorespiratory responses. More likely was that the CON exercise reduced muscle glycogen levels prior to the eccentric exercise. These novel findings are particularly relevant to individuals commencing a new training regimen or period, as well as those combining endurance and resistance exercise in rehabilitation programmes. It appears undergoing a bout of muscle-damaging exercise with reduced muscle glycogen availability can raise fat utilisation, even for low exercise intensity, as short as $12 \mathrm{~h}$ later. In athletic cohorts this raised fat utilisation may be seen when incorporating plyometric and/or sprint sessions into habitual training (be it aerobic- or anaerobic-based). Whereas in recreational athletes, it may occur without a substantial eccentric component, but still be glycogen depleting (if long prolonged) leading to muscle damage. For example, when performing recreational endurance events (such as cycle sportives) or during adventure vacations (such as cross-country hiking or skiing).

The design may inform future research examining how combined, prior concentric and eccentric exercise can be used to optimise fat metabolism in sedentary and athletic populations.

As part of a larger study on the systemic neuromuscular effect of pre-fatiguing exercise on eccentric contractions, the within-subject design 
negated i) comparison with a control group (eccentric exercise only), and ii) low-intensity exercise immediately after eccentric exercise (potential to influence muscle damage). However, low-intensity cycling at baseline and $12 \mathrm{~h}$ responses demonstrate that our protocol altered metabolic responses. Our next study aims to introduce a control group to determine the impact of concentric-biased exercise on metabolic alterations during the time-course of exerciseinduced muscle damage.

\section{Acknowledgements}

This study would not have been possible without our participants' commitment, time and effort. The study was supported by the laboratory technicians of the Department of Sport and Exercise Sciences (University of Chichester); all authors were involved from the project conception, to manuscript preparation.

\section{References}

Ahmaidi S, Granier P, Taoutaou Z, Mercier J, Dubouchaud H, Prefaut C. Effects of active recovery on plasma lactate and anaerobic power following repeated intensive exercise. Med Sci Sports Exerc, 1996; 28: 450456

Asp S, Daugaard JR, Kristiansen S, Kiens B, Richter EA. Exercise metabolism in human skeletal muscle exposed to prior eccentric exercise. J Physiol, 1998; 509: 305-313

Black CD, Dobson RM. Prior eccentric exercise reduces VO2peak and ventilatory threshold but does not alter movement economy during cycling exercise. J Strength Cond Res, 2012; 26: 2530-2537

Braun WA, Dutto DJ. The effects of a single bout of downhill running and ensuing delayed onset of muscle soreness on running economy performed $48 \mathrm{~h}$ later. Eur J Appl Physiol, 2003; 90: 29-34

Byrne C, Eston RG, Edwards RH. Characteristics of isometric and dynamic strength loss following eccentric exercise-induced muscle damage. Scand J Med Sci Sports, 2001; 11: 134-140

Calbet JA, Chavarren J, Dorado C. Running economy and delayed onset muscle soreness. J Sports Med Phys Fitness, 2001; 41: 18-26

Chen TC, Nosaka K, Tu JH. Changes in running economy following downhill running. J Sports Sci, 2007; 25: 55-63

Davies RC, Eston RG, Poole DC, Rowlands AV, DiMenna F, Wilkerson DP, Twist C, Jones J. Effect of eccentric exercise-induced muscle damage on the dynamics of muscle oxygenation and pulmonary oxygen uptake. J Appl Physiol, 2008; 105: 1413-1421

Davies RC, Rowlands AV, Eston RG. Effect of exercise-induced muscle damage on ventilatory and perceived exertion responses to moderate and severe intensity cycle exercise. Eur J Appl Physiol, 2009; 107: 11-19

Doncaster GG, Twist C. Exercise-induced muscle damage from bench press exercise impairs arm cranking endurance performance. Eur J Appl Physiol, 2012; 112: 4135-4142

Faul F, Erdfelder E, Lang AG, Buchner A. G*Power 3: a flexible statistical power analysis program for the social, behavioral, and biomedical sciences. Behav Res Methods, 2007; 39: 175-191

Gavin JP, Myers SD, Willems ME. Neuromuscular responses to mild-muscle damaging eccentric exercise in a low glycogen state. J Electromyogr Kinesiol, 2015; 25: 53-60

Gleeson M, Blannin AK, Zhu B, Brooks S, Cave R. Cardiorespiratory, hormonal and haematological responses to submaximal cycling performed 2 days after eccentric or concentric exercise bouts. J Sports Sci, 1995; 13: 471-479

Haouzi P, Chenuel B, Huszczuk A. Sensing vascular distension in skeletal muscle by slow conducting afferent fibers: neurophysiological basis and implication for respiratory control. J Appl Physiol, 2004; 96: 407-418

Heil DP, Wilcox AR, Quinn CM. Cardiorespiratory responses to seat-tube angle variation during steadystate cycling. Med Sci Sports Exerc, 1995; 27: 730-735

Jeukendrup AE, Wallis GA. Measurement of substrate oxidation during exercise by means of gas exchange measurements. Int J Sports Med, 2005; 26: S28-37 
Kano Y, Padilla DJ, Behnke BJ, Hageman KS, Musch TI, Poole DC. Effects of eccentric exercise on microcirculation and microvascular oxygen pressures in rat spinotrapezius muscle. J Appl Physiol, 2005; 99: 1516-1522

Kirwan JP, Hickner RC, Yarasheski KE, Kohrt WM, Wiethop BV, Holloszy JO. Eccentric exercise induces transient insulin resistance in healthy individuals. J Appl Physiol, 1992; 72: 2197-2202

Kyrolainen H, Pullinen T, Candau R, Avela J, Huttunen P, Komi PV. Effects of marathon running on running economy and kinematics. Eur J Appl Physiol, 2000; 82: 297-304

Lima-Silva AE, De-Oliveira FR, Nakamura FY, Gevaerd MS. Effect of carbohydrate availability on time to exhaustion in exercise performed at two different intensities. Braz J Med Biol Res, 2009; 42: 404-412

Marcora SM, Bosio A. Effect of exercise-induced muscle damage on endurance running performance in humans. Scand J Med Sci Sports, 2007; 17: 662-671

Molina R, Denadai BS. Muscle damage slows oxygen uptake kinetics during moderate-intensity exercise performed at high pedal rate. Appl Physiol Nutr Metab, 2011; 36: 848-855

Osborne MA, Schneider DA. Muscle glycogen reduction in man: relationship between surface EMG activity and oxygen uptake kinetics during heavy exercise. Exp Physiol, 2006; 91: 179-189

Paulsen G, Mikkelsen UR, Raastad T, Peake JM. Leucocytes, cytokines and satellite cells: what role do they play in muscle damage and regeneration following eccentric exercise? Exerc Immunol Rev, 2012; 18: 4297

Pernow B, Saltin B. Availability of substrates and capacity for prolonged heavy exercise in man. J Appl Physiol, 1971; 31: 416-422

Pilegaard H, Keller C, Steensberg A, Helge JW, Pedersen BK, Saltin B, Neufer PD. Influence of pre-exercise muscle glycogen content on exercise-induced transcriptional regulation of metabolic genes. J Physiol, 2002; 541: 261-271

Romijn JA, Coyle EF, Sidossis LS, Gastaldelli A, Horowitz JF, Endert E, Wolfe RR. Regulation of endogenous fat and carbohydrate metabolism in relation to exercise intensity and duration. Am J Physiol, 1993; 265: E380-391

Sargeant AJ, Dolan P. Human muscle function following prolonged eccentric exercise. Eur J Appl Physiol Occup Physiol, 1987; 56: 704-711

Schneider DA, Berwick JP, Sabapathy S, Minahan CL. Delayed onset muscle soreness does not alter O2 uptake kinetics during heavy-intensity cycling in humans. Int J Sports Med, 2007; 28: 550-556

Steensberg A, van Hall G, Keller C, Osada T, Schjerling P, Pedersen BK, Saltin B, Febbraio MA. Muscle glycogen content and glucose uptake during exercise in humans: influence of prior exercise and dietary manipulation. J Physiol, 2002; 541: 273-281

Suzuki M, Umeda T, Nakaji S, Shimoyama T, Mashiko T, Sugawara K. Effect of incorporating low intensity exercise into the recovery period after a rugby match. Br J Sports Med, 2004; 38: 436-440

Tuominen JA, Ebeling P, Bourey R, Koranyi L, Lamminen A, Rapola J, Sane T, Vuorinen-Markkola H, Koivisto VA. Postmarathon paradox: insulin resistance in the face of glycogen depletion. Am J Physiol, 1996; 270: E336-343

Twist C, Eston RG. The effect of exercise-induced muscle damage on perceived exertion and cycling endurance performance. Eur J Appl Physiol, 2009; 105: 559-567

Yamanaka R, Yunoki T, Arimitsu T, Lian CS, Roghayyeh A, Matsuura R, Yano T. Relationship between effort sense and ventilatory response to intense exercise performed with reduced muscle glycogen. Eur J Appl Physiol, 2012; 112: 2149-2162

\section{Corresponding author:}

James Peter Gavin

Department of Sport and Physical Activity, Bournemouth University, Fern Barrow

Poole, Dorset, United Kingdom

BH12 5BB

Phone: +44 (0)1202 566303 ; E-mail: jgavin@bournemouth.ac.uk 